\title{
On Intuitionistic Fuzzy Filters of Intuitionistic Fuzzy Coframes
}

\author{
Rajesh K. Thumbakara \\ Department of Mathematics, Mar Athanasius College, Kothamangalam, Kerala 686666, India \\ Correspondence should be addressed to Rajesh K. Thumbakara; rthumbakara@gmail.com
}

Received 28 January 2013; Accepted 11 March 2013

Academic Editor: Krassimir T. Atanassov

Copyright (C) 2013 Rajesh K. Thumbakara. This is an open access article distributed under the Creative Commons Attribution License, which permits unrestricted use, distribution, and reproduction in any medium, provided the original work is properly cited.

Frame theory is the study of topology based on its open set lattice, and it was studied extensively by various authors. In this paper, we study quotients of intuitionistic fuzzy filters of an intuitionistic fuzzy coframe. The quotients of intuitionistic fuzzy filters are shown to be filters of the given intuitionistic fuzzy coframe. It is shown that the collection of all intuitionistic fuzzy filters of a coframe and the collection of all intutionistic fuzzy quotient filters of an intuitionistic fuzzy filter are coframes.

\section{Introduction}

Frame theory is topology seen through notions of lattice theory; here one takes the lattice of open sets as the basic notion. The concept of Frames has been studied by many mathematicians including Banaschewski, Dowker, and Johnstone. For details one can refer to [1-3]. In 1965 Zadeh [4] introduced the concept of fuzzy sets as the generalization of ordinary subsets. The concept of ideals of a fuzzy subring was studied by Mordeson and Malik in [5] and Prajapati in [6]. In 1983, Atanassov [7] proposed a generalization of the notion of fuzzy set, known as intuitionistic fuzzy sets. In our earlier paper $[8,9]$, we introduced the concept of intuitionistic fuzzy frames and intuitionistic fuzzy filters of a frame. In this paper, intuitionistic fuzzy filters of a coframe and intuitionistic fuzzy filters of an intuitionistic fuzzy coframe are studied and examined. Subcoframe of the collection of all intuitionistic fuzzy filters of the coframe $F$ is obtained.

\section{Preliminaries}

In this section we will review some fundamental definitions.

Definition 1. Let $F$ be any set; then for any arbitrary $S \subseteq F$, $\wedge S=\inf \{x \mid x \in S\}$ and $\vee S=\sup \{x \mid x \in S\}$.

Definition 2 (see $[1,2]$ ). A frame is a complete lattice $F$ satisfying the infinite distributive law $a \wedge(\vee S)=\vee\{a \wedge x \mid$ $x \in S\}$ for any $a \in F$ and $S \subseteq F$.
Definition 3 (see $[1,2]$ ). A coframe is a complete lattice $F$ satisfying the infinite distributive law $a \vee(\wedge S)=\wedge\{a \vee x \mid$ $x \in S\}$ for any $a \in F$ and $S \subseteq F$.

Definition 4 (see $[1,2])$. A subset $M$ of a coframe $F$ closed under arbitrary meets, finite joins and having unit (top) element $e_{F}$ and zero (bottom) element $o_{F}$ of the coframe $F$ is called a sub coframe of $F$.

Definition 5 (see $[1,2])$. For any coframe $F$, a subset $P$ of $F$ is a filter if $x, y \in P \Rightarrow x \wedge y \in P$ and $x \in P, y \geq x \Rightarrow y \in P$.

Definition 6 (see [7]). Let $X$ be a nonempty set. An intuitionistic fuzzy set (IFS) $A$ of $X$ is an object of the form $A=\left\{\left(x, \mu_{A}(x), \gamma_{A}(x)\right) \mid x \in F\right\}$, where $\mu_{A}: X \rightarrow[0,1]$ and $\gamma_{A}: X \rightarrow[0,1]$ define, respectively, the degree of membership and the degree of nonmembership of the element $x \in X$ and $0 \leq \mu_{A}(x)+\gamma_{A}(x) \leq 1$, for all $x \in X$.

Definition 7 (see [8]). Let $F$ be a coframe then an IFS $A=$ $\left\{\left(x, \mu_{A}(x), \gamma_{A}(x)\right) \mid x \in F\right\}$ in $F$ is called an intuitionistic fuzzy coframe (IFCF) of $F$ if it satisfies the following conditions:

$$
\begin{aligned}
\text { (i) } \mu_{A}(x \vee y) & \geq \min \left\{\mu_{A}(x), \mu_{A}(y)\right\} \\
& \gamma_{A}(x \vee y) \leq \max \left\{\gamma_{A}(x), \gamma_{A}(y)\right\} \text { for all } x, y \in F, \\
\text { (ii) } & \mu_{A}(\wedge S) \geq \inf \left\{\mu_{A}(x) \mid x \in S\right\} \\
& \gamma_{A}(\wedge S) \leq \sup \left\{\gamma_{A}(x) \mid x \in S\right\} \text { for arbitrary } S \subseteq F,
\end{aligned}
$$


(iii) $\mu_{A}\left(e_{F}\right)=\mu_{A}\left(o_{F}\right) \geq \mu_{A}(x)$

$\gamma_{A}\left(e_{F}\right)=\gamma_{A}\left(o_{F}\right) \leq \gamma_{A}(x)$ for all $x \in F$, where $e_{F}$ and $o_{F}$ are, respectively, the unit and zero element of the coframe $F$.

Definition 8 (see [7]). If $\left\{A_{\alpha} \mid \alpha \in \Lambda\right\}$ is a family of IFS of $F$ where $A_{\alpha}=\left\{\left(x, \mu_{A_{\alpha}}(x), \gamma_{A_{\alpha}}(x)\right) \mid x \in F\right\}$, then

$$
\begin{aligned}
\bigcap_{\alpha \in \Lambda} A_{\alpha} & =\left\{\left(x, \mu_{\bigcap_{\alpha \in \Lambda} A_{\alpha}}(x), \gamma_{\bigcap_{\alpha \in \Lambda} A_{\alpha}}(x)\right) \mid x \in F\right\} \\
& =\left\{\left(x, \inf \left\{\mu_{A_{\alpha}}(x)\right\}, \sup \left\{\gamma_{A_{\alpha}}(x)\right\}\right) \mid x \in F\right\}, \\
\bigcup_{\alpha \in \Lambda} A_{\alpha} & =\left\{\left(x, \mu_{\bigcup_{\alpha \in \Lambda} A_{\alpha}}(x), \gamma_{\alpha \in \Lambda} A_{\alpha}(x)\right) \mid x \in F\right\} \\
& =\left\{\left(x, \sup \left\{\mu_{A_{\alpha}}(x)\right\}, \inf \left\{\gamma_{A_{\alpha}}(x)\right\}\right) \mid x \in F\right\} .
\end{aligned}
$$

Definition 9 (see [9]). The operation of meet $\wedge$ and join $\vee$ on a coframe $F$ can be extended to operations $\widetilde{\Lambda}$ and $\widetilde{V}$ on the set of all intuitionistic fuzzy set IFS of $F$ as follows.

For $A, B \in$ IFS one has

$$
A \tilde{\wedge} B=\left\{\left(x, \mu_{A \tilde{\wedge} B}(x), \gamma_{A \tilde{\wedge} B}(x)\right) \mid x \in F\right\},
$$

where $\mu_{A \tilde{\wedge} B}(x)=\sup _{u \wedge v=x}\left\{\mu_{A}(u) \wedge \mu_{B}(v)\right\}$ and $\gamma_{A \tilde{\wedge} B}(x)=$ $\inf _{u \wedge v=x}\left\{\gamma_{A}(u) \vee \gamma_{B}(v)\right\}$

$$
A \widetilde{\nabla} B=\left\{\left(x, \mu_{A \widetilde{V} B}(x), \gamma_{A \widetilde{V} B}(x)\right) \mid x \in F\right\},
$$

where $\mu_{A \widetilde{v} B}(x)=\sup _{u \vee v=x}\left\{\mu_{A}(u) \wedge \mu_{B}(v)\right\}$ and $\gamma_{A \widetilde{v} B}(x)=$ $\inf _{u \vee v=x}\left\{\gamma_{A}(u) \vee \gamma_{B}(v)\right\}$.

The original operation $\wedge$ and $\vee$ on a coframe $F$ can be retrieved from $\widetilde{\wedge}$ and $\widetilde{V}$ by embedding $F$ into IFS as the set of all intuitionistic fuzzy singletons each of which is an IFS,

$$
1_{x}=\left\{\left(y, \mu_{x}(y), \gamma_{x}(y)\right) \mid y \in F\right\}
$$

where

$$
\begin{aligned}
& \mu_{x}(y)= \begin{cases}1 & \text { if } y=x, \\
0 & \text { otherwise }\end{cases} \\
& \gamma_{x}(y)= \begin{cases}0 & \text { if } y=x \\
1 & \text { otherwise }\end{cases}
\end{aligned}
$$

Lemma 10 (see [9]). Let $A, B, C \in \mathrm{IFS}$; then

(i) $A \widetilde{\vee}(B \tilde{\wedge} C) \subseteq(A \widetilde{\vee} B) \tilde{\wedge}(A \widetilde{\vee} C)$

(ii) $A \subseteq B \Rightarrow A \widetilde{\vee} C \subseteq B \widetilde{\vee} C$.

\section{Intuitionistic Fuzzy Filter of a Coframe}

In this section, we show that the collection of all intuitionistic fuzzy filters of the coframe $F$ is a coframe. We use $\operatorname{IFF}(F)$ to denote intuitionistic fuzzy filter of the coframe $F$.
Definition 11 (see [9]). Let $F$ be a coframe; then an IFS $A$ on $F$ is said to be an $\operatorname{IFF}(F)$ if

(F1) $\mu_{A}(x \wedge y) \geq \min \left\{\mu_{A}(x), \mu_{A}(y)\right\}$, for all $x, y \in F$ $\gamma_{A}(x \wedge y) \leq \max \left\{\gamma_{A}(x), \gamma_{A}(y)\right\}$, for all $x, y \in F$,

(F2) $\mu_{\mathrm{A}}(x \vee y) \geq \max \left\{\mu_{A}(x), \mu_{A}(y)\right\}$, for all $x, y \in F$ $\gamma_{A}(x \vee y) \leq \min \left\{\gamma_{A}(x), \gamma_{A}(y)\right\}$, for all $x, y \in F$,

(F3) $\mu_{A}\left(e_{F}\right)=1, \gamma_{A}\left(e_{F}\right)=0$ where $e_{F}$ is the unit element of $F$.

Lemma 12 (see [9]). If $A$ and $B$ are any two $\operatorname{IFF}(F)$, then, $A \cap B$ is an $\operatorname{IFF}(F)$.

Result 1 (see [9]). Union of any two $\operatorname{IFF}(F)$ needs not be an $\operatorname{IFF}(F)$.

Definition 13. Let $A$ be an IFS. Let $\langle A\rangle=\bigcap\{B \mid A \subseteq B$, $B$ an $\operatorname{IFF}(F)\}$ where $A \subseteq B$ means $\mu_{A}(x) \leq \mu_{B}(x)$ and $\gamma_{A}(x) \geq$ $\gamma_{B}(x)$ for all $x \in F$. Then $\langle A\rangle$ is called the $\operatorname{IFF}(F)$ generated by $A$.

Theorem 14. Let $A, B$ be two $\operatorname{IFF}(F)$; then $A \tilde{\wedge} B$ is an $\operatorname{IFF}(F)$ and $A \tilde{\wedge} B=\langle A \cup B\rangle$.

Proof. Let $x \in F$; then

$$
\begin{aligned}
& (A \tilde{\wedge} B)(x) \\
& =\left\{\left(x, \sup _{u \wedge v=x}\left\{\mu_{A}(u) \wedge \mu_{B}(v)\right\}, \inf _{u \wedge v=x}\left\{\gamma_{A}(u) \vee \gamma_{B}(v)\right\}\right) \mid\right. \\
& \quad x \in F\} \\
& \supseteq\left(x, \mu_{A}(x) \wedge \mu_{B}\left(e_{F}\right), \gamma_{A}(x) \vee \gamma_{B}\left(e_{F}\right)\right) \\
& =\left(x, \mu_{A}(x), \gamma_{A}(x)\right)=A .
\end{aligned}
$$

Hence $A \widetilde{\wedge} B \supseteq A$. Similarly, we have $A \widetilde{\wedge} B \supseteq$ B. Thus, $A \widetilde{\wedge} B \supseteq$ $A \cup B$. Also,

$$
\begin{aligned}
& \mu_{A \tilde{\wedge} B}(x \wedge y) \\
& =\sup _{u \wedge v=x \wedge y}\left\{\mu_{A}(u) \wedge \mu_{B}(v)\right\} \\
& \geq \bigvee\left\{\mu_{A}\left(u_{1} \wedge v_{1}\right) \wedge \mu_{B}\left(u_{2} \wedge v_{2}\right) \mid\right. \\
& \left.\quad u_{1} \wedge u_{2}=x, v_{1} \wedge v_{2}=y ; u_{1}, v_{1}, u_{2}, v_{2} \in F\right\} \\
& \geq \bigvee\left\{\left(\mu_{A}\left(u_{1}\right) \wedge \mu_{A}\left(v_{1}\right)\right) \wedge\left(\mu_{B}\left(u_{2}\right) \wedge \mu_{B}\left(v_{2}\right)\right) \mid\right. \\
& \left.u_{1} \wedge u_{2}=x, v_{1} \wedge v_{2}=y ; u_{1}, v_{1}, u_{2}, v_{2} \in F\right\}
\end{aligned}
$$




$$
\begin{aligned}
& =\bigvee\left\{\left(\mu_{A}\left(u_{1}\right) \wedge \mu_{B}\left(u_{2}\right)\right) \wedge\left(\mu_{A}\left(v_{1}\right) \wedge \mu_{B}\left(v_{2}\right)\right) \mid\right. \\
& \left.u_{1} \wedge u_{2}=x, v_{1} \wedge v_{2}=y ; u_{1}, v_{1}, u_{2}, v_{2} \in F\right\} \\
& =\bigvee\left\{\mu_{A}\left(u_{1}\right) \wedge \mu_{B}\left(u_{2}\right) \mid u_{1} \wedge u_{2}=x ; u_{1}, u_{2} \in F\right\} \\
& \wedge \bigvee\left\{\mu_{A}\left(v_{1}\right) \wedge \mu_{B}\left(v_{2}\right) \mid v_{1} \wedge v_{2}=y ; v_{1}, v_{2} \in F\right\} \\
& =\mu_{A \widetilde{\wedge} B}(x) \wedge \mu_{A \widetilde{\wedge} B}(y) \quad \forall x, y \in F, \\
& \gamma_{A \tilde{\wedge} B}(x \wedge y) \\
& =\inf _{u \wedge v=x \wedge y}\left\{\gamma_{A}(u) \vee \gamma_{B}(v)\right\} \\
& \leq \bigwedge\left\{\gamma_{A}\left(u_{1} \wedge v_{1}\right) \vee \gamma_{B}\left(u_{2} \wedge v_{2}\right) \mid\right. \\
& \left.u_{1} \wedge u_{2}=x, v_{1} \wedge v_{2}=y ; u_{1}, v_{1}, u_{2}, v_{2} \in F\right\} \\
& \leq \bigwedge\left\{\left(\gamma_{A}\left(u_{1}\right) \vee \gamma_{A}\left(v_{1}\right)\right) \vee\left(\gamma_{B}\left(u_{2}\right) \vee \gamma_{B}\left(v_{2}\right)\right) \mid\right. \\
& \left.u_{1} \wedge u_{2}=x, v_{1} \wedge v_{2}=y ; u_{1}, v_{1}, u_{2}, v_{2} \in F\right\} \\
& =\bigwedge\left\{\left(\gamma_{A}\left(u_{1}\right) \vee \gamma_{B}\left(u_{2}\right)\right) \vee\left(\gamma_{A}\left(v_{1}\right) \vee \gamma_{B}\left(v_{2}\right)\right) \mid\right. \\
& \left.u_{1} \wedge u_{2}=x, v_{1} \wedge v_{2}=y ; u_{1}, v_{1}, u_{2}, v_{2} \in F\right\} \\
& =\bigwedge\left\{\gamma_{A}\left(u_{1}\right) \vee \gamma_{B}\left(u_{2}\right) \mid u_{1} \wedge u_{2}=x ; u_{1}, u_{2} \in F\right\} \\
& \vee \bigwedge\left\{\gamma_{A}\left(v_{1}\right) \vee \gamma_{B}\left(v_{2}\right) \mid v_{1} \wedge v_{2}=y ; v_{1}, v_{2} \in F\right\} \\
& =\gamma_{A \tilde{\wedge} B}(x) \vee \gamma_{A \tilde{\wedge} B}(y) \quad \forall x, y \in F,
\end{aligned}
$$

(F2)

$$
\begin{aligned}
& \mu_{A \tilde{\wedge} B}(x \vee y) \\
& =\sup _{u \wedge v=x \vee y}\left\{\mu_{A}(u) \wedge \mu_{B}(v)\right\} \\
& \geq \bigvee\left\{\mu_{A}(y \vee u) \wedge \mu_{B}(y \vee v) \mid u \wedge v=x ; u, v \in F\right\} \\
& {[\because(y \vee u) \wedge(y \vee v)=x \vee y]} \\
& \geq \bigvee\left\{\mu_{A}(u) \wedge \mu_{B}(v) \mid u \wedge v=x ; u, v \in F\right\} \\
& =\mu_{A \tilde{\wedge} B}(x) \quad \forall x, y \in F .
\end{aligned}
$$

Similarly, we have $\mu_{A \widetilde{\wedge} B}(x \vee y) \geq \mu_{A \tilde{\wedge} B}(y)$ for all $x, y \in F$.
F

Therefore, $\mu_{A \tilde{\wedge} B}(x \vee y) \geq \mu_{A \tilde{\wedge} B}(x) \vee \mu_{A \tilde{\wedge} B}(y)$, for all $x, y \in$

$$
\begin{aligned}
& \gamma_{A \tilde{\wedge} B}(x \vee y) \\
& =\inf _{u \wedge v=x \vee y}\left\{\gamma_{A}(u) \vee \gamma_{B}(v)\right\} \\
& \leq \bigwedge\left\{\gamma_{A}(y \vee u) \vee \gamma_{B}(y \vee v) \mid u \wedge v=x ; u, v \in F\right\} \\
& \qquad[\because(y \vee u) \wedge(y \vee v)=x \vee y] \\
& \leq \bigwedge\left\{\gamma_{A}(u) \vee \gamma_{B}(v) \mid u \wedge v=x ; u, v \in F\right\} \\
& =\gamma_{A \tilde{\wedge} B}(x) \quad \forall x, y \in F .
\end{aligned}
$$

Similarly, we have $\gamma_{A \widetilde{\wedge} B}(x \vee y) \leq \gamma_{A \tilde{\wedge} B}(y)$ for all $x, y \in F$. F.

Therefore, $\gamma_{A \tilde{\wedge} B}(x \vee y) \leq \gamma_{A \tilde{\wedge} B}(x) \wedge \gamma_{A \tilde{\wedge} B}(y)$, for all $x, y \in$

(F3) Since $\mu_{A}\left(e_{F}\right)=\mu_{B}\left(e_{F}\right)=1$, clearly $\mu_{A \widetilde{\wedge} B}\left(e_{F}\right)=$ $\sup _{u \wedge v=e_{F}}\left\{\mu_{A}(u) \wedge \mu_{B}(v)\right\} \geq \mu_{A}\left(e_{F}\right) \wedge \mu_{B}\left(e_{F}\right)=1$ and since $\gamma_{A}\left(e_{F}\right)=\gamma_{B}\left(e_{F}\right)=0$, we have $\gamma_{A \widetilde{\wedge} B}\left(e_{F}\right)=$ $\inf _{u \wedge v=e_{F}}\left\{\gamma_{A}(u) \wedge \gamma_{B}(v)\right\} \leq \gamma_{A}\left(e_{F}\right) \wedge \gamma_{B}\left(e_{F}\right)=0$.

Thus if $A, B$ are $\operatorname{IFF}(F)$, then $A \tilde{\wedge} B$ is an $\operatorname{IFF}(F)$.

Now let $C$ be any $\operatorname{IFF}(F)$ such that $A \cup B \subseteq C$, then,

$$
\begin{aligned}
\mu_{A \tilde{\wedge} B}(x) & =\sup _{u \wedge v=x}\left\{\mu_{A}(u) \wedge \mu_{B}(v)\right\} \\
& \leq \sup _{u \wedge v=x}\left\{\mu_{C}(u) \wedge \mu_{C}(v)\right\} \\
& \leq \sup _{u \wedge v=x}\left\{\mu_{C}(u \wedge v)\right\} \\
& =\mu_{C}(x), \\
\gamma_{A \tilde{\wedge} B}(x) & =\inf _{u \wedge v=x}\left\{\gamma_{A}(u) \vee \gamma_{B}(v)\right\} \\
& \geq \inf _{u \wedge v=x}\left\{\gamma_{C}(u) \vee \gamma_{C}(v)\right\} \\
& \geq \inf _{u \wedge v=x}\left\{\gamma_{C}(u \wedge v)\right\} \\
& =\gamma_{C}(x) .
\end{aligned}
$$

Hence $A \tilde{\wedge} B \subseteq C$. Thus $A \tilde{\wedge} B$ is the smallest filter of $F$ such that $A \cup B \subseteq A \tilde{\wedge} B$.

Hence $A \widetilde{\wedge} B=\langle A \cup B\rangle$.

Proposition 15. Let $A, B$ be two $\operatorname{IFF}(F)$; then $A \widetilde{\nabla} B=A \cap B$.

Proof. We have, for $x \in F, \mu_{A \widetilde{v} B}(x)=\sup _{u \vee v=x}\left\{\mu_{A}(u) \wedge\right.$ $\left.\mu_{B}(v)\right\} \geq \mu_{A}(x) \wedge \mu_{B}(x)=\mu_{A \cap B}(x)$ and $\gamma_{A \widetilde{\nabla} B}(x)=$ $\inf _{u \vee v=x}\left\{\gamma_{A}(u) \vee \gamma_{B}(v)\right\} \leq \gamma_{A}(x) \vee \gamma_{B}(x)=\gamma_{A \cap B}(x)$. Hence $A \cap B \subseteq A \widetilde{\vee} B$. 
Now since $\mu_{i}(u \vee v)=\mu_{i}(x) \geq \mu_{i}(u) \vee \mu_{i}(v)$ and hence $\mu_{i}(x) \geq \mu_{i}(u)$ and $\mu_{i}(x) \geq \mu_{i}(v)$ for $i=A, B$

$$
\begin{aligned}
\mu_{A \widetilde{\nabla} B}(x) & =\sup _{u \vee v=x}\left\{\mu_{A}(u) \wedge \mu_{B}(v)\right\} \\
& \leq \sup _{u \vee v=x}\left\{\mu_{A}(x) \wedge \mu_{B}(x)\right\} \\
& =\mu_{A}(x) \wedge \mu_{B}(x)=\mu_{A \cap B}(x), \\
\gamma_{A \widetilde{\vee} B}(x) & =\inf _{u \vee v=x}\left\{\gamma_{A}(u) \vee \gamma_{B}(v)\right\} \\
& \geq \inf _{u \vee v=x}\left\{\gamma_{A}(x) \vee \gamma_{B}(x)\right\} \\
& =\gamma_{A}(x) \vee \gamma_{B}(x)=\gamma_{A \cap B}(x) .
\end{aligned}
$$

Hence $A \widetilde{\nabla} B \subseteq A \cap B$. Therefore $A \widetilde{\nabla} B=A \cap B$.

Theorem 16. Given an arbitrary collection $\left(A_{\alpha}\right)_{\alpha \in \wedge}$ and $A$ of $\operatorname{IFF}(F)$, then

$$
A \cap\left(\bigwedge_{\alpha \in \Lambda} A_{\alpha}\right)=\bigwedge_{\alpha \in \Lambda}\left(A \cap A_{\alpha}\right) .
$$

Proof. We have for $w \in F$,

$$
\begin{aligned}
& \mu_{A \cap\left(\tilde{\wedge}_{\alpha \in \wedge} A_{\alpha}\right)}(w)=\mu_{A}(w) \wedge\left(\bigwedge_{\alpha \in \wedge} \mu_{A_{\alpha}}\right)(w) \\
& =\mu_{A}(w) \wedge \sup _{\wedge_{\alpha \in \wedge} a_{\alpha}=w}\left\{\bigwedge_{\alpha \in \wedge} \mu_{A_{\alpha}}\left(a_{\alpha}\right)\right\} \\
& =\sup _{\wedge_{\alpha \in \wedge} a_{\alpha}=w}\left\{\bigwedge_{\alpha \in \Lambda}\left(\mu_{A}(w) \wedge \mu_{A_{\alpha}}\left(a_{\alpha}\right)\right)\right\} \\
& \leq \sup _{\wedge \in \wedge_{\alpha}=w}\left\{\bigwedge_{\alpha \in \Lambda}\left(\mu_{A}\left(a_{\alpha} \vee w\right) \wedge \mu_{A_{\alpha}}\left(a_{\alpha}\right)\right)\right\} \\
& =\sup _{\wedge_{\alpha \in \wedge} a_{\alpha}=w}\left\{\bigwedge_{\alpha \in \wedge}\left(\mu_{A}\left(a_{\alpha}\right) \wedge \mu_{A_{\alpha}}\left(a_{\alpha}\right)\right)\right\} \\
& =\sup _{\wedge_{\alpha \in \wedge} a_{\alpha}=w}\left\{\bigwedge_{\alpha \in \wedge}\left(\mu_{A} \cap \mu_{A_{\alpha}}\right)\left(a_{\alpha}\right)\right\} \\
& =\mu_{\tilde{\Lambda}_{\alpha \in \wedge}\left(A \cap A_{\alpha}\right)}(w), \gamma_{A \cap\left(\tilde{\Lambda}_{\alpha \in \wedge} A_{\alpha}\right)}(w) \\
& =\gamma_{A}(w) \wedge\left(\bigwedge_{\alpha \in \wedge} \gamma_{A_{\alpha}}\right)(w) \\
& =\gamma_{A}(w) \wedge \inf _{\wedge_{\alpha \in \wedge} a_{\alpha}=w}\left\{\bigvee_{\alpha \in \wedge} \gamma_{A_{\alpha}}\left(a_{\alpha}\right)\right\} \\
& =\inf _{\wedge_{\alpha \in \wedge} a_{\alpha}=w}\left\{\bigvee_{\alpha \in \wedge}\left(\gamma_{A}(w) \wedge \gamma_{A_{\alpha}}\left(a_{\alpha}\right)\right)\right\} \\
& \geq \inf _{\wedge_{\alpha \in \wedge} a_{\alpha}=w}\left\{\bigvee_{\alpha \in \wedge}\left(\gamma_{A}\left(a_{\alpha} \vee w\right) \wedge \gamma_{A_{\alpha}}\left(a_{\alpha}\right)\right)\right\} \\
& =\inf _{\wedge_{\alpha \in \wedge} a_{\alpha}=w}\left\{\bigvee_{\alpha \in \wedge}\left(\gamma_{A}\left(a_{\alpha}\right) \wedge \gamma_{A_{\alpha}}\left(a_{\alpha}\right)\right)\right\}
\end{aligned}
$$

$$
\begin{aligned}
& =\inf _{\wedge_{\alpha \in \wedge} a_{\alpha}=w}\left\{\bigvee_{\alpha \in \wedge}\left(\gamma_{A} \cap \gamma_{A_{\alpha}}\right)\left(a_{\alpha}\right)\right\} \\
& =\gamma_{\tilde{\Lambda}_{\alpha \in \wedge}\left(A \cap A_{\alpha}\right)}(w) .
\end{aligned}
$$

Therefore,

$$
A \cap\left(\bigwedge_{\alpha \in \wedge} A_{\alpha}\right) \subseteq \bigwedge_{\alpha \in \Lambda}\left(A \cap A_{\alpha}\right) .
$$

Also $A \cap A_{\alpha} \subseteq A_{\alpha}$ and $A \cap A_{\alpha} \subseteq A$ for all $\alpha \in \wedge$; hence $\widetilde{\Lambda}_{\alpha \in \wedge}\left(A \cap A_{\alpha}\right) \subseteq \widetilde{\bigwedge}_{\alpha \in \wedge} A_{\alpha}$ and $\widetilde{\bigwedge}_{\alpha \in \wedge}\left(A \cap A_{\alpha}\right) \subseteq A$. Therefore,

$$
\bigwedge_{\alpha \in \Lambda}\left(A \cap A_{\alpha}\right) \subseteq A \cap\left(\bigwedge_{\alpha \in \Lambda} A_{\alpha}\right) .
$$

From (14) and (15) we have $\widetilde{\bigwedge}_{\alpha \in \wedge}\left(A \cap A_{\alpha}\right)=A \cap\left(\widetilde{\bigwedge}_{\alpha \in \wedge} A_{\alpha}\right)$.

Remark 17. If we interchange the roles of $\tilde{\Lambda}$ and $\cap$ in Theorem 16, only one-sided inequality $A \tilde{\wedge}\left(\bigcap_{\alpha \in \wedge} A_{\alpha}\right) \subseteq$ $\bigcap_{\alpha \in \wedge}\left(A \tilde{\wedge} A_{\alpha}\right)$ holds.

Theorem 18. The set $I_{F} F$ of all intuitionistic fuzzy filters of the coframe $F$ is a coframe.

Proof. $I_{F} F$ is a complete lattice, which is bounded above by $A_{\chi_{F}}=\left\{\left(x, \mu_{A_{\chi_{F}}}(x), \gamma_{A_{\chi_{F}}}(x)\right) \mid x \in F\right\}$ where $\mu_{A_{\chi_{F}}}(x)=1$, $\gamma_{A_{\chi_{F}}}(x)=0$ for all $x \in F$ and bounded below by $A_{e_{F}}=\{(x$, $\left.\left.\mu_{e_{F}}(x), \gamma_{e_{F}}(x)\right) \mid x \in F\right\}$ where

$$
\begin{array}{r}
\mu_{e_{F}}(x)= \begin{cases}1 & x=e_{F}, \\
0 & \text { otherwise }\end{cases} \\
\gamma_{e_{F}}(x)= \begin{cases}0 & x=e_{F} \\
1 & \text { otherwise }\end{cases} \\
\forall x \in F .
\end{array}
$$

Also $\widetilde{\bigwedge}_{\alpha \in \Lambda}\left(A \widetilde{V} A_{\alpha}\right)=A \widetilde{V}\left(\widetilde{\bigwedge}_{\alpha \in \Lambda} A_{\alpha}\right)$ for arbitrary collection $\left(A_{\alpha}\right)_{\alpha \in \wedge}$ and $A$ of $\operatorname{IFF}(F)$ by Proposition 15 and Theorem 16. Hence $I_{F} F$ is a coframe.

\section{Intuitionistic Fuzzy Filter of an Intuitionistic Fuzzy Coframe}

Definition 19. Let $A$ be an intuitionistic fuzzy coframe (IFCF) of $F$ and $B$ an IFS with $B \subseteq A$. Then $B$ is called an intuitionistic fuzzy filter of $A(\operatorname{IFF}(A))$ if

(i) $\mu_{B}(x \wedge y) \geq \min \left\{\mu_{B}(x), \mu_{B}(y)\right\}$ $\gamma_{B}(x \wedge y) \leq \max \left\{\gamma_{B}(x), \gamma_{B}(y)\right\}$.

(ii) $\mu_{B}(x \vee y) \geq \max \left\{\mu_{A}(x) \wedge \mu_{B}(y), \mu_{B}(x) \wedge \mu_{A}(y)\right\}$ for all $x, y \in F$. $\gamma_{B}(x \vee y) \leq \min \left\{\gamma_{A}(x) \vee \gamma_{B}(y), \gamma_{B}(x) \vee \gamma_{A}(y)\right\}$ for all $x, y \in F$. 
(iii) $\mu_{B}\left(e_{F}\right)=\mu_{A}\left(e_{F}\right)$ and $\gamma_{B}\left(e_{F}\right)=\gamma_{A}\left(e_{F}\right)$ where $e_{F}$ is the unit element of $F$.

If $B$ is an $\operatorname{IFF}(A)$, then we write $B \triangleleft A$.

Theorem 20. Let $A$ be an IFCF of $F$ and $B$ an $\operatorname{IFF}(A)$. Then $A \cap B$ is an $\operatorname{IFF}(A)$.

Proof. Obviously $A \cap B \subseteq A$ and

(i)

$$
\begin{aligned}
\mu_{A \cap B}(x \wedge y) \\
=\min \left\{\mu_{A}(x \wedge y), \mu_{B}(x \wedge y)\right\} \\
\geq \min \left\{\min \left(\mu_{A}(x), \mu_{A}(y)\right), \min \left(\mu_{B}(x), \mu_{B}(y)\right)\right\} \\
\geq \min \left\{\min \left(\mu_{A}(x), \mu_{B}(x)\right), \min \left(\mu_{A}(y), \mu_{B}(y)\right)\right\} \\
=\mu_{A \cap B}(x) \wedge \mu_{A \cap B}(y), \quad \forall x, y \in F, \\
\gamma_{A \cap B}(x \wedge y) \\
=\max \left\{\gamma_{A}(x \wedge y), \gamma_{B}(x \wedge y)\right\} \\
\quad \leq \max \left\{\max \left(\gamma_{A}(x), \gamma_{A}(y)\right), \max \left(\gamma_{B}(x), \gamma_{B}(y)\right)\right\} \\
=\max \left\{\max \left(\gamma_{A}(x), \gamma_{B}(x)\right), \max \left(\gamma_{A}(y), \gamma_{B}(y)\right)\right\} \\
=\gamma_{A \cap B}(x) \vee \gamma_{A \cap B}(y),
\end{aligned}
$$$$
\forall x, y \in F
$$

(ii)

$$
\begin{aligned}
\mu_{A \cap B} & (x \vee y) \\
= & \min \left\{\mu_{A}(x \vee y), \mu_{B}(x \vee y)\right\} \\
\geq & \min \left\{\mu_{A}(x) \wedge \mu_{A}(y),\right. \\
& \left.\quad\left(\mu_{A}(x) \wedge \mu_{B}(y)\right) \vee\left(\mu_{B}(x) \wedge \mu_{A}(y)\right)\right\} \\
= & \left(\mu_{A}(x) \wedge \mu_{A}(y)\right) \\
& \wedge\left[\left(\mu_{A}(x) \wedge \mu_{B}(y)\right) \vee\left(\mu_{B}(x) \wedge \mu_{A}(y)\right)\right] \\
= & \left(\mu_{A}(x) \wedge \mu_{A}(y) \wedge \mu_{B}(y)\right) \\
& \vee\left(\mu_{A}(x) \wedge \mu_{B}(x) \wedge \mu_{A}(y)\right) \\
= & \left(\mu_{A}(x) \wedge \mu_{A \cap B}(y)\right) \\
& \vee\left(\mu_{A \cap B}(x) \wedge \mu_{A}(y)\right)
\end{aligned}
$$

$\forall x, y \in F$,

$$
\begin{aligned}
& \gamma_{A \cap B}(x \vee y) \\
& =\max \left\{\gamma_{A}(x \vee y), \gamma_{B}(x \vee y)\right\} \\
& \quad \leq \max \left\{\gamma_{A}(x) \vee \gamma_{A}(y),\right.
\end{aligned}
$$$$
\left.\left(\gamma_{A}(x) \vee \gamma_{B}(y)\right) \wedge\left(\gamma_{B}(x) \vee \gamma_{A}(y)\right)\right\}
$$

$$
\begin{aligned}
&=\left(\gamma_{A}(x) \vee \gamma_{A}(y)\right) \\
& \vee\left[\left(\gamma_{A}(x) \vee \gamma_{B}(y)\right) \wedge\left(\gamma_{B}(x) \vee \gamma_{A}(y)\right)\right] \\
&=\left(\gamma_{A}(x) \vee \gamma_{A}(y) \vee \gamma_{B}(y)\right) \\
& \wedge\left(\gamma_{A}(x) \vee \gamma_{B}(x) \vee \gamma_{A}(y)\right) \\
&=\left(\gamma_{A}(x) \vee \gamma_{A \cap B}(y)\right) \wedge\left(\gamma_{A \cap B}(x) \vee \gamma_{A}(y)\right) \\
& \forall x, y \in F,
\end{aligned}
$$

(iii) also clearly $\mu_{A \cap B}\left(e_{F}\right)=\mu_{A}\left(e_{F}\right)$ and $\gamma_{A \cap B}\left(e_{F}\right)=\gamma_{A}\left(e_{F}\right)$ where $e_{F}$ is the unit element of $F$.

Theorem 21. Let $A$ be an IFCF of $F$, and let $B, C$ be two $\operatorname{IFF}(A)$. Then $B \cap C$ is also an $\operatorname{IFF}(A)$.

Proof. Clearly $B \cap C \subseteq A$ also

(i)

$$
\begin{array}{r}
\mu_{B \cap C}(x \wedge y) \geq \mu_{B \cap C}(x) \wedge \mu_{B \cap C}(y), \\
\gamma_{A \cap B}(x \wedge y) \leq \gamma_{A \cap B}(x) \vee \\
\gamma_{A \cap B}(y) \\
\forall x, y \in F,
\end{array}
$$

(ii)

$$
\begin{aligned}
& \mu_{B \cap C}(x \vee y) \\
&= \min \left\{\mu_{B}(x \vee y), \mu_{C}(x \vee y)\right\} \\
& \geq \min \left\{\left(\mu_{A}(x) \wedge \mu_{B}(y)\right) \vee\left(\mu_{B}(x) \wedge \mu_{A}(y)\right),\right. \\
&\left.\left(\mu_{A}(x) \wedge \mu_{C}(y)\right) \vee\left(\mu_{C}(x) \wedge \mu_{A}(y)\right)\right\} \\
&= {\left[\mu_{A}(x) \wedge \mu_{B}(y) \vee\left(\mu_{B}(x) \wedge \mu_{A}(y)\right)\right] } \\
& \wedge\left[\left(\mu_{A}(x) \wedge \mu_{C}(y)\right) \vee\left(\mu_{C}(x) \wedge \mu_{A}(y)\right)\right] \\
& \geq\left(\mu_{A}(x) \wedge \mu_{B}(y) \wedge \mu_{C}(y)\right) \vee\left(\mu_{B}(x) \wedge \mu_{C}(x) \wedge \mu_{A}(y)\right) \\
&= {\left[\mu_{A}(x) \wedge \mu_{B \cap C}(y)\right] \vee\left[\mu_{B \cap C}(x) \wedge \mu_{A}(y)\right] } \\
& \gamma_{B \cap C}(x \vee y) \quad \forall x, y \in F, \\
&= \max \left\{\gamma_{B}(x \vee y), \gamma_{C}(x \vee y)\right\} \\
& \leq \max \left\{\left(\gamma_{A}(x) \vee \gamma_{B}(y)\right) \wedge\left(\gamma_{B}(x) \vee \gamma_{A}(y)\right),\right. \\
&\left.\quad\left(\gamma_{A}(x) \vee \gamma_{C}(y)\right) \wedge\left(\gamma_{C}(x) \vee \gamma_{A}(y)\right)\right\} \\
&=\left[\left(\gamma_{A}(x) \vee \gamma_{B}(y)\right) \wedge\left(\gamma_{B}(x) \vee \gamma_{A}(y)\right)\right] \\
& \vee\left[\left(\gamma_{A}(x) \vee \gamma_{C}(y)\right) \wedge\left(\gamma_{C}(x) \vee \gamma_{A}(y)\right)\right] \\
& \leq\left(\gamma_{A}(x) \vee \gamma_{A}(y) \vee \gamma_{B}(y)\right) \wedge\left(\gamma_{A}(x) \vee \gamma_{B}(x) \vee \gamma_{A}(y)\right) \\
&=\left(\gamma_{A}(x) \vee \gamma_{A \cap B}(y)\right) \vee\left(\gamma_{A \cap B}(x) \vee \gamma_{A}(y)\right)
\end{aligned}
$$

(iii) also $\mu_{B \cap C}\left(e_{F}\right)=\mu_{A}\left(e_{F}\right)$ and $\gamma_{B \cap C}\left(e_{F}\right)=\gamma_{A}\left(e_{F}\right)$. 
Theorem 22. Let $A$ be an IFCF of F and $B$ an IFS with $B \subseteq A$. Then $B$ is an $\operatorname{IFF}(A)$ if and only if

(i) $\mu_{B}(x \wedge y) \geq \mu_{B}(x) \wedge \mu_{B}(y) ; \gamma_{B}(x \wedge y) \leq \gamma_{B}(x) \vee \gamma_{B}(y)$ for all $x, y \in F$,

(ii) $A \widetilde{\vee} B \subseteq B$,

(iii) $\mu_{B}\left(e_{F}\right)=\mu_{A}\left(e_{F}\right)$ and $\gamma_{B}\left(e_{F}\right)=\gamma_{A}\left(e_{F}\right)$.

Proof. Suppose conditions (i), (ii), and (iii) holds to prove that $B$ is an $\operatorname{IFF}(A)$.

Since $A \widetilde{\vee} B \subseteq B$, one has

$$
\begin{aligned}
\mu_{B}(x \vee y) & \geq \mu_{A \widetilde{\vee} B}(x \vee y) \\
& =\sup _{u \vee v=x \vee y}\left\{\mu_{A}(u) \wedge \mu_{B}(v)\right\} \\
& \geq \mu_{A}(x) \wedge \mu_{B}(y) \quad \forall x, y \in F, \\
\gamma_{B}(x \vee y) & \leq \gamma_{A \widetilde{\vee} B}(x \vee y) \\
& =\inf _{u \vee v=x \vee y}\left\{\gamma_{A}(u) \vee \gamma_{B}(v)\right\} \\
& \leq \gamma_{A}(x) \wedge \gamma_{B}(y) \quad \forall x, y \in F .
\end{aligned}
$$

Also $\mu_{B}(x \vee y) \geq \mu_{A}(y) \wedge \mu_{B}(x)$ and $\gamma_{B}(x \vee y) \leq \gamma_{A}(y) \wedge \gamma_{B}(x)$ for all $x, y \in F$.

Hence,

$$
\begin{aligned}
& \mu_{B}(x \vee y) \geq \max \left\{\mu_{A}(x) \wedge \mu_{B}(y), \mu_{B}(x) \wedge \mu_{A}(y)\right\}, \\
& \gamma_{B}(x \vee y) \leq \min \left\{\gamma_{A}(x) \vee \gamma_{B}(y), \gamma_{B}(x) \vee \gamma_{A}(y)\right\} .
\end{aligned}
$$

Therefore $B$ is an $\operatorname{IFF}(A)$.

Conversely if $B$ is an $\operatorname{IFF}(A)$, we have $\mu_{B}(x \wedge y) \geq$ $\min \left\{\mu_{B}(x), \mu_{B}(y)\right\}, \gamma_{B}(x \wedge y) \leq \max \left\{\gamma_{B}(x), \gamma_{B}(y)\right\}$, and $\mu_{B}\left(e_{F}\right)=\mu_{A}\left(e_{F}\right), \gamma_{B}\left(e_{F}\right)=\gamma_{A}\left(e_{F}\right)$ from Definition 19.

Now for all $z \in F$ with $z=x \vee y, \mu_{B}(z) \geq$ $\sup _{x \vee y=z}\left\{\mu_{A}(x) \wedge \mu_{B}(y)\right\}=\mu_{A \widetilde{\vee} B}(z)$ and $\gamma_{B}(z) \leq$ $\inf _{x \vee y=z}\left\{\gamma_{A}(x) \vee \gamma_{B}(y)\right\}=\gamma_{A \widetilde{\vee} B}(z)$. Hence $A \widetilde{\vee} B \subseteq B$.

Theorem 23. Let $A$ be an IFCF of $F$, and let $B, C$ be two $\operatorname{IFF}(A)$; then $B \tilde{\wedge} C$ is an $\operatorname{IFF}(A)$ and $B \subseteq B \tilde{\wedge} C, C \subseteq B \tilde{\wedge} C$.

Proof. (i) We have $\mu_{B \widetilde{\wedge} C}(x \wedge y) \geq \mu_{B \widetilde{\wedge} C}(x) \wedge \mu_{B \tilde{\wedge} C}(y)$ and $\gamma_{B \tilde{\wedge} C}(x \wedge y) \leq \gamma_{B \tilde{\wedge} C}(x) \wedge \gamma_{B \tilde{\wedge} C}(y)$ from the proof of Theorem 14.

(ii) Now by Theorem 22 since $B, C$ are $\operatorname{IFF}(A)$, we have $A \widetilde{\vee} B \subseteq B$ and $A \widetilde{\vee} C \subseteq C$.

Hence $A \widetilde{\nabla}(B \tilde{\wedge} C) \subseteq(A \widetilde{\nabla} B) \tilde{\wedge}(A \widetilde{\vee} C) \subseteq B \widetilde{\wedge} C$ by Lemma 10.

(iii) Also clearly $\mu_{B \tilde{\wedge} C}\left(e_{F}\right)=\mu_{A}\left(e_{F}\right)$ and $\gamma_{B \tilde{\wedge} C}\left(e_{F}\right)=$ $\gamma_{A}\left(e_{F}\right)$.

Hence $B \widetilde{\wedge} C$ is an $\operatorname{IFF}(A)$ by Theorem 22 .

Again $\mu_{B \tilde{\wedge} C}(x)=\sup _{u \wedge v=x}\left\{\mu_{B}(u) \wedge \mu_{C}(v)\right\} \geq \mu_{B}(x) \wedge$ $\mu_{C}\left(e_{F}\right)=\mu_{B}(x)$ and $\gamma_{B \tilde{\wedge} C}(x)=\inf _{u \wedge v=x}\left\{\gamma_{B}(u) \vee \gamma_{C}(v)\right\} \leq$ $\gamma_{B}(x) \vee \gamma_{C}\left(e_{F}\right)=\gamma_{B}(x)$ for every $x \in F$.

Hence $B \subseteq B \widetilde{\wedge} C$. Similarly $C \subseteq B \widetilde{\wedge} C$.

\section{Quotient of Intuitionistic Fuzzy Filters of the Intuitionistic Fuzzy Coframe}

Definition 24. Let $A$ be an IFCF of $F$, and let $B, C$ be $\operatorname{IFF}(A)$. Then the quotient of $B$ by $C$ is denoted as $[B: C]$ and is defined as

$$
[B: C]=\bigcup\{D \mid D \triangleleft A, D \widetilde{\vee} C \subseteq B\}
$$

The collection of all quotients of any intuitionistic fuzzy filter $B$ of $A$ is denoted by $[B]_{A}$.

Theorem 25. Let $A$ be an IFCF of $F$, and let $B, C$ be $\operatorname{IFF}(A)$. Then $[B: C]$ is an $\operatorname{IFF}(A)$. Also $B \subseteq[B: C] \subseteq A$.

Proof. Let $\widetilde{A}=\{D \mid D \triangleleft A$ and $D \widetilde{\nabla} C \subseteq B\}$. Suppose $D, D^{\prime} \in \widetilde{A}$, then $D$ and $D^{\prime}$ are $\operatorname{IFF}(A)$ such that $D \widetilde{V} C \subseteq B$ and $D^{\prime} \widetilde{\nabla} C \subseteq B$. Now by Theorem $23 D \widetilde{\wedge} D^{\prime}$ is an $\operatorname{IFF}(A)$.

Now $\left(D \tilde{\wedge} D^{\prime}\right) \widetilde{V} C \subseteq(D \widetilde{\vee} C) \widetilde{\wedge}\left(D^{\prime} \widetilde{\vee} C\right) \subseteq B \widetilde{\wedge} B \subseteq B$ by Lemma 10 and since

$$
\begin{aligned}
\mu_{B \tilde{\wedge} B}(x) & =\sup _{u \wedge v=x}\left\{\mu_{B}(u) \wedge \mu_{B}(v)\right\} \\
& \leq \sup _{u \wedge v=x} \mu_{B}(u \wedge v)=\mu_{B}(x), \\
\gamma_{B \tilde{\wedge} B}(x) & =\inf _{u \wedge v=x}\left\{\gamma_{B}(u) \vee \gamma_{B}(v)\right\} \\
& \geq \gamma_{B}(u \wedge v)=\gamma_{B}(x) .
\end{aligned}
$$

Hence $D \tilde{\wedge} D^{\prime} \in \widetilde{A}$.

Now

$$
\begin{aligned}
\mu_{[B: C]}(x) \wedge \mu_{[B: C]}(y) & =\left(\bigvee_{D \in \widetilde{A}} \mu_{D}(x)\right) \wedge\left(\bigvee_{D^{\prime} \in \tilde{A}} \mu_{D^{\prime}}(y)\right) \\
& =\bigvee_{D, D^{\prime} \in \widetilde{A}}\left\{\mu_{D}(x) \wedge \mu_{D^{\prime}}(y)\right\} \\
& \leq \bigvee_{D, D^{\prime} \in \widetilde{A}} \mu_{D \tilde{\wedge} D^{\prime}}(x \wedge y) \\
& \leq \mu_{[B: C]}(x \wedge y),
\end{aligned}
$$

also

$$
\begin{aligned}
\gamma_{[B: C]}(x) \vee \gamma_{[B: C]}(y) & =\left(\bigwedge_{D \in \widetilde{A}} \gamma_{D}(x)\right) \vee\left(\bigwedge_{D^{\prime} \in \widetilde{A}} \gamma_{D^{\prime}}(y)\right) \\
& =\bigwedge_{D, D^{\prime} \in \widetilde{A}}\left\{\gamma_{D}(x) \vee \gamma_{D^{\prime}}(y)\right\} \\
& \geq \bigwedge_{D, D^{\prime} \in \widetilde{A}} \gamma_{D \tilde{\wedge} D^{\prime}}(x \wedge y) \\
& \geq \gamma_{[B: C]}(x \wedge y),
\end{aligned}
$$

since $D \tilde{\wedge} D^{\prime} \in \widetilde{A}$. 
Hence

$$
\begin{aligned}
& \mu_{[B: C]}(x \wedge y) \geq \min \left\{\mu_{[B: C]}(x), \mu_{[B: C]}(y)\right\} \\
& \gamma_{[B: C]}(x \wedge y) \leq \max \left\{\gamma_{[B: C]}(x), \gamma_{[B: C]}(y)\right\}
\end{aligned}
$$

Now

$$
\begin{aligned}
\mu_{[B: C]}(x \vee y) & =\bigvee_{D \in \widetilde{A}} \mu_{D}(x \vee y) \\
& \geq \bigvee_{D \in \widetilde{A}}\left\{\left(\mu_{A}(x) \wedge \mu_{D}(y)\right)\right. \\
& \left.\vee\left(\mu_{D}(x) \wedge \mu_{A}(y)\right)\right\} \\
\geq & \bigvee_{D \in \widetilde{A}}\left(\mu_{A}(x) \wedge \mu_{D}(y)\right) \\
= & \mu_{A}(x) \wedge\left\{\underset{D \in \widetilde{A}}{\left.\bigvee_{D}\left(\mu_{D}(y)\right)\right\}}\right. \\
= & \mu_{A}(x) \wedge \mu_{[B: C]}(y) .
\end{aligned}
$$

Similarly $\mu_{[B: C]}(x \vee y) \geq \mu_{[B: C]}(x) \wedge \mu_{A}(y)$.

Thus

$$
\mu_{[B: C]}(x \vee y) \geq \max \left\{\mu_{A}(x) \wedge \mu_{[B: C]}(y), \mu_{[B: C]}(x) \wedge \mu_{A}(y)\right\}
$$

Now

$$
\begin{aligned}
\gamma_{[B: C]}(x \vee y)= & \bigwedge_{D \in \widetilde{A}} \gamma_{D}(x \vee y) \\
\leq & \bigwedge_{D \in \widetilde{A}}\left\{\left(\gamma_{A}(x) \vee \gamma_{D}(y)\right)\right. \\
& \left.\wedge\left(\gamma_{D}(x) \vee \gamma_{A}(y)\right)\right\} \\
\leq & \bigwedge_{D \in \widetilde{A}}\left(\gamma_{A}(x) \vee \gamma_{D}(y)\right) \\
= & \gamma_{A}(x) \vee\left\{\bigwedge_{D \in \widetilde{A}} \gamma_{D}(y)\right\} \\
= & \gamma_{A}(x) \vee \gamma_{[B: C]}(y) .
\end{aligned}
$$

Similarly $\gamma_{[B: C]}(x \vee y) \leq \gamma_{[B: C]}(x) \vee \gamma_{A}(y)$.

Hence

$$
\begin{array}{r}
\gamma_{[B: C]}(x \vee y) \leq \min \left\{\gamma_{A}(x) \vee \gamma_{[B: C]}(y), \gamma_{[B: C]}(x) \vee \gamma_{A}(y)\right\} \\
\forall x, y \in F .
\end{array}
$$

Also

$$
\begin{aligned}
& \mu_{[B: C]}\left(e_{F}\right)=\bigvee_{D \in \widetilde{A}} \mu_{D}\left(e_{F}\right)=\mu_{A}\left(e_{F}\right), \\
& \gamma_{[B: C]}\left(e_{F}\right)=\bigwedge_{D \in \widetilde{A}} \gamma_{D}\left(e_{F}\right)=\gamma_{A}\left(e_{F}\right) .
\end{aligned}
$$

Now from (27), (29), (31), and (32), [B:C] is an $\operatorname{IFF}(A)$.

Also clearly $[B: C] \subseteq A$. Since $B$ is an $\operatorname{IFF}(A)$, we have by Theorem 22, $A \widetilde{\mathrm{V}} B \subseteq B$.

Now by Lemma 10 since $C \subseteq A$, we have $C \widetilde{\mathrm{V}} B \subseteq A \widetilde{\mathrm{V}} B \subseteq$ $B$. Hence $B \in \widetilde{A}$ and so $B \subseteq[B: C]$. Thus $B \subseteq[B: C] \subseteq A$.

Theorem 26. Let $A$ be an IFCF of F, and let $B, C, E$ be $\operatorname{IFF}(A)$. Then the following holds:

(1) if $B \subseteq C$; then $[B: E] \subseteq[C: E]$ and $[E: C] \subseteq[E: B]$,

(2) if $B \subseteq C$; then $[C: B]=A$,

(3) $[B: B]=A$,

(4) $[B: C]=[B: B \tilde{\wedge} C]$.

Proof. (1) Let $B \subseteq C$. Let $\widetilde{A}=\{D \mid D \triangleleft A$ and $D \widetilde{V} E \subseteq B\}$ and $\widetilde{A}=\{D \mid D \triangleleft A$ and $D \widetilde{V} E \subseteq C\}$.

If $D \in \widetilde{A}$, then $D \triangleleft A$ and $D \widetilde{\vee} E \subseteq B \subseteq C$. Therefore $D \in \widetilde{A}$ and hence $\widetilde{A} \subseteq \widetilde{A}^{\prime}$.

So $[B: E]=\bigcup_{D \in \widetilde{A}} D \subseteq \bigcup_{D \in \widetilde{A}^{\prime}} D=[C: E]$ and hence $[B: E] \subseteq[C: E]$.

Similarly it can be shown that $[E: C] \subseteq[E: B]$.

(2) Let $\widetilde{A}=\{D \mid D \triangleleft A$ and $D \widetilde{\vee} B \subseteq C\}$. We have $A \triangleleft A$. Also $B \triangleleft A$ implies that $A \widetilde{\vee} B \subseteq B \subseteq C$.

Hence $A \in \widetilde{A}$, and so $A \subseteq \bigcup_{D \in \widetilde{A}} D=[C: B]$. Also since $[C: B]$ is an $\operatorname{IFF}(A),[C: B] \subseteq A$.

Therefore, $[C: B]=A$.

(3) We have $B \subseteq B$. So from Theorem 26(2) we have [ $B$ : $B]=A$.

(4) We have by Theorem $23 B \tilde{\wedge} C$ is an $\operatorname{IFF}(A)$ and $C \subseteq$ $B \tilde{\wedge} C$.

Hence $[B: B \tilde{\wedge} C] \subseteq[B: C]$ from Theorem 26(1).

Let $\widetilde{A}=\{D \mid D \triangleleft A$ and $D \widetilde{V} C \subseteq B\}$ and $\widetilde{A}=\{D \mid D \triangleleft$ $A$ and $D \widetilde{\vee}(B \widetilde{\wedge} C) \subseteq B\}$.

If $D \in \widetilde{A}$, then $D \triangleleft A$ and $D \widetilde{\vee} C \subseteq B$. Now since $D \subseteq A$, $D \widetilde{\vee} B \subseteq A \widetilde{\vee} B$ by Lemma 10 .

Also by Theorem 22, $B \triangleleft A$ implies $A \widetilde{\nabla} B \subseteq B$. Hence $D \widetilde{\vee} B \subseteq B$.

Therefore, by Lemma 10, $D \widetilde{\nabla}(B \widetilde{\wedge} C) \subseteq(D \widetilde{\vee} B) \widetilde{\wedge}(D \widetilde{V}$ $C) \subseteq B \widetilde{\wedge} B=B$ and hence $D \in \widetilde{A}$.

So $[B: C]=\bigcup_{D \in \widetilde{A}} D \subseteq \bigcup_{D \in \widetilde{A}^{\prime}} D=[B: B \widetilde{\wedge} C]$. Thus $[B: B \tilde{\wedge} C]=[B: C]$.

Corollary 27. Let $A$ be an $\operatorname{IFCF}$ of $F$, and let $B, C$ be $\operatorname{IFF}(A)$. Then

(1) $[B: B \cap C]=A$,

(2) $[B \tilde{\wedge} C: B \cap C]=A$,

(3) $[[B: B]: C]=A$. 
Proof. (1) Since $B \cap C$ is an $\operatorname{IFF}(A)$ by Theorem 20 and $B \cap C \subseteq$ $B$, we have $[B: B \cap C]=A$ by Theorem 26 .

(2) Since $B \cap C \subseteq B \tilde{\wedge} C$, we have $[B \tilde{\wedge} C: B \cap C]=A$ by Theorem 26.

(3) By Theorem 26, $[B: B]=A$ also $C \subseteq A$. Hence $[[B$ : $B]: C]=A$ by Theorem 26 .

Theorem 28. Let $A$ be an IFCF of F, and let $B, C, E$ be $\operatorname{IFF}(A)$. Then $[B: C \tilde{\wedge} E]=[B: C] \cap[B: E]$.

Proof. We have $C \tilde{\wedge} E$ is an $\operatorname{IFF}(A)$ such that $C \subseteq C \tilde{\wedge} E$ and $E \subseteq C \widetilde{\wedge} E$ by Theorem 23 .

Hence $[B: C \tilde{\wedge} E] \subseteq[B: C]$ and $[B: C \tilde{\wedge} E] \subseteq[B: E]$ by Theorem 26.

Hence

$$
[B: C \tilde{\wedge} E] \subseteq[B: C] \cap[B: E] .
$$

Let $\widetilde{A}=\{D \mid D \triangleleft A$ and $D \widetilde{V} C \subseteq B\}, \widetilde{A}^{\prime}=\{D \mid D \triangleleft$ $A$ and $D \widetilde{\nabla} E \subseteq B\}$ and $\widetilde{A}^{\prime \prime}=\{D \mid D \triangleleft A$ and $D \widetilde{\nabla}(C \widetilde{\wedge} E) \subseteq$ $B\}$.

For every $x \in F$,

$$
\begin{aligned}
\mu_{[B: C]}[B: E] & (x) \\
= & \mu_{[B: C]}(x) \wedge \mu_{[B: E]}(x) \\
= & \left(\bigvee_{D \in \widetilde{A}} \mu_{D}(x)\right) \wedge\left(\bigvee_{D^{\prime} \in \widetilde{A}^{\prime}} \mu_{D^{\prime}}(x)\right) \\
= & \left.\bigvee_{D^{\prime} \in \widetilde{A}^{\prime}}\left(\bigvee_{D \in \widetilde{A}} \mu_{D}(x)\right) \wedge \mu_{D^{\prime}}(x)\right) \\
= & \bigvee_{D^{\prime} \in \widetilde{A}^{\prime}}\left(\bigvee_{D \in \widetilde{A}}\left(\mu_{D}(x) \wedge \mu_{D^{\prime}}(x)\right)\right) \\
= & \bigvee_{D, D^{\prime}}\left\{\mu_{D}(x) \wedge \mu_{D^{\prime}}(x) \mid D \in \widetilde{A}, D^{\prime} \in \widetilde{A}^{\prime}\right\},
\end{aligned}
$$

also

$$
\begin{aligned}
\gamma_{[B: C] \cap[B: E]}(x) & \\
= & \gamma_{[B: C]}(x) \vee \gamma_{[B: E]}(x) \\
& \left.=\bigwedge_{D \in \widetilde{A}} \gamma_{D}(x)\right) \vee\left(\bigwedge_{D^{\prime} \in \widetilde{A}^{\prime}} \gamma_{D^{\prime}}(x)\right) \\
& \left.=\bigwedge_{D^{\prime} \in \widetilde{A}^{\prime}}\left(\bigwedge_{D \in \widetilde{A}} \gamma_{D}(x)\right) \vee \gamma_{D^{\prime}}(x)\right) \\
& =\bigwedge_{D^{\prime} \in \widetilde{A^{\prime}}}\left(\bigwedge_{D \in \widetilde{A}}\left(\gamma_{D}(x) \vee \gamma_{D^{\prime}}(x)\right)\right) \\
= & \bigwedge_{D, D^{\prime}}\left\{\gamma_{D}(x) \vee \gamma_{D^{\prime}}(x) \mid D \in \widetilde{A}, D^{\prime} \in \widetilde{A}^{\prime}\right\} .
\end{aligned}
$$

Let $D \in \widetilde{A}, D^{\prime} \in \widetilde{A}$; then $D, D^{\prime}$ are $\operatorname{IFF}(A)$ and $D \widetilde{\nabla} C \subseteq B$, $D^{\prime} \widetilde{\vee} E \subseteq B$.

Now by Theorem $21 D \cap D^{\prime}$ is an $\operatorname{IFF}(A)$, and also

$$
\begin{aligned}
(D \cap & \left.D^{\prime}\right) \widetilde{\nabla}(C \tilde{\wedge} E) \\
& \subseteq\left(\left(D \cap D^{\prime}\right) \widetilde{\nabla} C\right) \tilde{\wedge}\left(\left(D \cap D^{\prime}\right) \widetilde{\nabla} E\right) \\
& \subseteq(D \widetilde{\nabla} C) \widetilde{\wedge}\left(D^{\prime} \widetilde{\nabla} E\right) \subseteq B \widetilde{\wedge} B=B .
\end{aligned}
$$

Hence $D \cap D^{\prime} \in \widetilde{A}^{\prime \prime}$. Thus

$$
[B: C \tilde{\wedge} E]=\bigcup_{D \in A^{\prime \prime}} D \supseteq \bigcup_{D, D^{\prime}}\left\{D \cap D^{\prime} \mid D \in \widetilde{A}, D^{\prime} \in \widetilde{A}^{\prime}\right\} .
$$

Now

$$
\begin{aligned}
\mu_{[B: C \tilde{\wedge} E]}(x) & \geq \bigvee_{D, D^{\prime}}\left\{\mu_{D \cap D^{\prime}}(x) \mid D \in \widetilde{A}, D^{\prime} \in \widetilde{A}^{\prime}\right\} \\
& =\bigvee_{D, D^{\prime}}\left\{\mu_{D}(x) \wedge \mu_{D^{\prime}}(x) \mid D \in \widetilde{A}, D^{\prime} \in \widetilde{A}^{\prime}\right\} \\
& =\mu_{[B: C] \cap[B: E]}(x) .
\end{aligned}
$$

Also

$$
\begin{aligned}
\gamma_{[B: C \tilde{\wedge} E]}(x) & \leq \bigwedge_{D, D^{\prime}}\left\{\gamma_{D \cap D^{\prime}}(x) \mid D \in \widetilde{A}, D^{\prime} \in \widetilde{A}^{\prime}\right\} \\
& =\bigwedge_{D, D^{\prime}}\left\{\gamma_{D}(x) \vee \gamma_{D^{\prime}}(x) \mid D \in \widetilde{A}, D^{\prime} \in \widetilde{A}^{\prime}\right\} \\
& =\gamma_{[B: C] \cap[B: E]}(x)
\end{aligned}
$$

from (35).

Thus

$$
[B: C \widetilde{\wedge} E] \supseteq[B: C] \cap[B: E] .
$$

Consequently $[B: C \widetilde{\wedge} E]=[B: C] \cap[B: E]$ from (33) and (40).

Theorem 29. Let $A$ be an IFCF of F, and let the arbitrary collection $\left(A_{\alpha}\right)_{\alpha \in \wedge}, B$, and $C$ be $\operatorname{IFF}(A)$; then for the arbitrary collection $\left[B: A_{\alpha}\right]_{\alpha \in \wedge}$ and $[B: C]$ of quotients of $B$,

$$
[B: C] \cap\left(\bigwedge_{\alpha \in \wedge}\left[B: A_{\alpha}\right]\right)=\bigwedge_{\alpha \in \Lambda}\left([B: C] \cap\left[B: A_{\alpha}\right]\right) \text {. }
$$

Proof. We have

$$
\bigwedge_{\alpha \in \Lambda}\left([B: C] \cap\left[B: A_{\alpha}\right]\right)=\bigwedge_{\alpha \in \Lambda}\left[B: C \tilde{\wedge} A_{\alpha}\right] .
$$

By Theorem 28. 
Now from Theorem 23, $C \subseteq C \tilde{\wedge} A_{\alpha}$ and $A_{\alpha} \subseteq C \tilde{\wedge} A_{\alpha}$.

Therefore, by Theorem $26,\left[B: C \widetilde{\wedge} A_{\alpha}\right] \subseteq[B: C]$ and $\left[B: C \widetilde{\wedge} A_{\alpha}\right] \subseteq\left[B: A_{\alpha}\right]$.

Hence

$$
\begin{gathered}
\bigwedge_{\alpha \in \Lambda}\left[B: C \tilde{\wedge} A_{\alpha}\right] \subseteq[B: C], \\
\bigwedge_{\alpha \in \Lambda}\left[B: C \tilde{\wedge} A_{\alpha}\right] \subseteq \bigwedge_{\alpha \in \Lambda}\left[B: A_{\alpha}\right] .
\end{gathered}
$$

So,

$$
\bigwedge_{\alpha \in \wedge}\left[B: C \tilde{\wedge} A_{\alpha}\right] \subseteq[B: C] \cap\left(\bigwedge_{\alpha \in \wedge}\left[B: A_{\alpha}\right]\right) .
$$

Hence,

$$
\bigwedge_{\alpha \in \Lambda}\left([B: C] \cap\left[B: A_{\alpha}\right]\right) \subseteq[B: C] \cap\left(\bigwedge_{\alpha \in \wedge}\left[B: A_{\alpha}\right]\right)
$$

by (42).

Also for $x \in F$,

$$
\begin{aligned}
& \mu_{[B: C] \cap\left(\tilde{\lambda}_{\alpha \in \wedge}\left[B: A_{\alpha}\right]\right)}(x)
\end{aligned}
$$

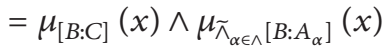

$$
\begin{aligned}
& =\mu_{[B: C]}(x) \wedge \sup \left\{\bigwedge_{\alpha \in \Lambda} \mu_{\left[B: A_{\alpha}\right]}\left(a_{\alpha}\right) \mid a_{\alpha} \in F, \bigwedge_{\alpha \in \Lambda} a_{\alpha}=x\right\} \\
& =\sup \left\{\bigwedge_{\alpha \in \Lambda}\left(\mu_{[B: C]}(x) \wedge \mu_{\left[B: A_{\alpha}\right]}\left(a_{\alpha}\right)\right) \mid a_{\alpha} \in F, \bigwedge_{\alpha \in \Lambda} a_{\alpha}=x\right\} \\
& =\sup \left\{\bigwedge_{\alpha \in \Lambda}\left(\mu_{[B: C]}(x) \wedge\left[\mu_{A}\left(a_{\alpha}\right) \wedge \mu_{\left[B: A_{\alpha}\right]}\left(a_{\alpha}\right)\right]\right) \mid\right. \\
& \left.a_{\alpha} \in F, \bigwedge_{\alpha \in \Lambda} a_{\alpha}=x\right\} \quad\left(\because\left[B: A_{\alpha}\right] \subseteq A\right) \\
& =\sup \left\{\bigwedge_{\alpha \in \Lambda}\left(\left[\mu_{[B: C]}(x) \wedge \mu_{A}\left(a_{\alpha}\right)\right] \wedge \mu_{\left[B: A_{\alpha}\right]}\left(a_{\alpha}\right)\right) \mid\right. \\
& \left.a_{\alpha} \in F, \bigwedge_{\alpha \in \Lambda} a_{\alpha}=x\right\} \\
& \leq \sup \left\{\bigwedge_{\alpha \in \Lambda}\left(\mu_{[B: C]}\left(a_{\alpha} \vee x\right) \wedge \mu_{\left[B: A_{\alpha}\right]}\left(a_{\alpha}\right)\right) \mid\right. \\
& \left.a_{\alpha} \in F, \bigwedge_{\alpha \in \Lambda} a_{\alpha}=x\right\} \quad(\because[B: C] \text { is an } \operatorname{IFF}(F)) \\
& =\sup \left\{\bigwedge_{\alpha \in \Lambda}\left(\mu_{[B: C]}\left(a_{\alpha}\right) \wedge \mu_{\left[B: A_{\alpha}\right]}\left(a_{\alpha}\right)\right) \mid a_{\alpha} \in F, \bigwedge_{\alpha \in \Lambda} a_{\alpha}=x\right\} \\
& =\sup \left\{\bigwedge_{\alpha \in \Lambda}\left(\mu_{[B: C] \cap\left[B: A_{\alpha}\right]}\left(a_{\alpha}\right)\right) \mid a_{\alpha} \in F, \bigwedge_{\alpha \in \Lambda} a_{\alpha}=x\right\} \\
& =\mu_{\tilde{\Lambda}_{\alpha \in \Lambda}\left([B: C] \cap\left[B: A_{\alpha}\right]\right)}(x) \text {, }
\end{aligned}
$$

$$
\begin{aligned}
& \gamma_{[B: C] \cap\left(\tilde{\lambda}_{\alpha \in \wedge}\left[B: A_{\alpha}\right]\right)}(x) \\
& =\gamma_{[B: C]}(x) \vee \gamma_{\kappa_{\alpha \in \Lambda}\left[B: A_{\alpha}\right]}(x) \\
& =\gamma_{[B: C]}(x) \vee \inf \left\{\bigvee_{\alpha \in \Lambda} \gamma_{\left[B: A_{\alpha}\right]}\left(a_{\alpha}\right) \mid a_{\alpha} \in F, \bigwedge_{\alpha \in \Lambda} a_{\alpha}=x\right\} \\
& =\inf \left\{\bigvee_{\alpha \in \Lambda}\left(\gamma_{[B: C]}(x) \vee \gamma_{\left[B: A_{\alpha}\right]}\left(a_{\alpha}\right)\right) \mid a_{\alpha} \in F, \bigwedge_{\alpha \in \Lambda} a_{\alpha}=x\right\} \\
& =\inf \left\{\bigvee_{\alpha \in \wedge}\left(\left(\gamma_{[B: C]}(x) \vee \gamma_{A}\left(a_{\alpha}\right)\right) \vee \gamma_{\left[B: A_{\alpha}\right]}\left(a_{\alpha}\right)\right) \mid\right. \\
& \left.a_{\alpha} \in F, \bigwedge_{\alpha \in \Lambda} a_{\alpha}=x\right\} \\
& \geq \inf \left\{\bigvee_{\alpha \in \Lambda}\left(\gamma_{[B: C]}\left(a_{\alpha} \vee x\right) \vee \gamma_{\left[B: A_{\alpha}\right]}\left(a_{\alpha}\right)\right) \mid\right. \\
& \left.a_{\alpha} \in F, \bigwedge_{\alpha \in \Lambda} a_{\alpha}=x\right\} \quad(\because[B: C] \text { is an } \operatorname{IFF}(F)) \\
& =\inf \left\{\bigvee_{\alpha \in \Lambda}\left(\gamma_{[B: C]}\left(a_{\alpha}\right) \vee \gamma_{\left[B: A_{\alpha}\right]}\left(a_{\alpha}\right)\right) \mid a_{\alpha} \in F, \bigwedge_{\alpha \in \Lambda} a_{\alpha}=x\right\} \\
& =\sup \left\{\bigvee_{\alpha \in \Lambda}\left(\mu_{[B: C] \cap\left[B: A_{\alpha}\right]}\left(a_{\alpha}\right)\right) \mid a_{\alpha} \in F, \bigwedge_{\alpha \in \Lambda} a_{\alpha}=x\right\} \\
& =\mu_{\tau_{\alpha \in \Lambda}\left([B: C] \cap\left[B: A_{\alpha}\right]\right)}(x) \text {. }
\end{aligned}
$$

Hence,

$$
[B: C] \cap\left(\bigwedge_{\alpha \in \Lambda}\left[B: A_{\alpha}\right]\right) \subseteq \bigwedge_{\alpha \in \Lambda}\left([B: C] \cap\left[B: A_{\alpha}\right]\right) .
$$

Therefore $[B: C] \cap\left(\widetilde{\bigwedge}_{\alpha \in \wedge}\left[B: A_{\alpha}\right]\right)=\widetilde{\bigwedge}_{\alpha \in \wedge}\left([B: C] \cap\left[B: A_{\alpha}\right]\right)$ from (45) and (47).

Remark 30. If we interchange the roles of $\widetilde{\Lambda}$ and $\bigcap$ in Theorem 29, only one-sided inequality $[B: C] \widetilde{\wedge}\left(\bigcap_{\alpha \in \wedge}[B\right.$ : $\left.\left.A_{\alpha}\right]\right) \subseteq \bigcap_{\alpha \in \wedge}\left([B: C] \widetilde{\wedge}\left[B: A_{\alpha}\right]\right)$ holds.

Theorem 31. Let $A$ be an IFCF of F; then the collection of all intuitionistic fuzzy quotient filter of any intuitionistic fuzzy filter $B$ of $A,[B]_{A}$ is a coframe.

Proof. $[B]_{A}$ is a complete lattice, which is bounded above by $A$ and below by $A_{e}=\left\{\left(x, \mu_{A_{e}}(x), \gamma_{A_{e}}(x)\right) \mid x \in F\right\}$ where

$$
\begin{aligned}
& \mu_{A_{e}}(x)= \begin{cases}\mu_{A}\left(e_{F}\right), & x=e_{F}, \\
0, & \text { otherwise },\end{cases} \\
& \gamma_{A_{e}}(x)= \begin{cases}\gamma_{A}\left(e_{F}\right), & x=e_{F}, \\
0, & \text { otherwise. }\end{cases}
\end{aligned}
$$

Also $[B: C] \widetilde{V}\left(\widetilde{\wedge}_{\alpha \in \wedge}\left[B: A_{\alpha}\right]\right)=\widetilde{\Lambda}_{\alpha \in \wedge}\left([B: C] \widetilde{V}\left[B: A_{\alpha}\right]\right)$ for arbitrary collection $\left(A_{\alpha}\right)_{\alpha \in \wedge}, B$ and $C$ of intuitionistic fuzzy filters of $A$ from Proposition 15 and Theorem 29.

Hence $[B]_{A}$ is a coframe. 
Theorem 32. The collection of all intuitionistic fuzzy quotient filters of any intuitionistic fuzzy filter $B$ of the intuitionistic fuzzy coframe $A_{\chi_{F}}$ of $F,[B]_{A_{\chi_{F}}}$ is the sub coframe of the collection of all intuitionistic fuzzy filters $I_{F} F$ of $F$, where $A_{\chi_{F}}=\{(x$, $\left.\left.\mu_{A_{\chi_{F}}}(x), \gamma_{A_{\chi_{F}}}(x)\right) \mid x \in F\right\}, \mu_{A_{\chi_{F}}}(x)=1, \gamma_{A_{\chi_{F}}}(x)=0$ for all $x \in F$.

Proof. Follows from Theorems 18 and 31 as $[B]_{A_{\chi_{F}}} \subseteq I_{F} F$. Also $[B: B]=A_{\chi_{F}}$ and $A_{e}=A_{e_{F}}$ are, respectively, top and bottom elements of $[B]_{A_{\chi_{F}}}$.

\section{Acknowledgments}

The author is thankful to Professor T. Thrivikraman, Former Head, Department of Mathematics, Cochin University of Science and Technology, Cochin, India. The author also acknowledges the financial assistance received from University Grants Commission, New Delhi.

\section{References}

[1] B. Banaschewski, Lectures on Frame, University of Cape Town, 1988.

[2] C. H. Dowker and D. Papert, "Quotient frames and subspaces," Proceedings of the London Mathematical Society, vol. 16, pp. 275296, 1966.

[3] P. T. Johnstone, Stone spaces, vol. 3 of Cambridge Studies in Advanced Mathematics, Cambridge University Press, Cambridge, UK, 1982.

[4] L. A. Zadeh, "Fuzzy sets," Information and Computation, vol. 8, pp. 338-353, 1965.

[5] J. N. Mordeson and D. S. Malik, Fuzzy Commutative Algebra, World Scientific Publishing, River Edge, NJ, USA, 1998, With a foreword by Azriel Rosenfeld.

[6] A. S. Prajapati, "Residual of ideals of an L-ring," Iranian Journal of Fuzzy Systems, vol. 4, no. 2, pp. 69-82, 2007.

[7] K. T. Atanassov, "Intuitionistic fuzzy sets," in VII ITKR's Session, Sofia, V. Sgurev, Ed., Central Science and Technical Library, Bulgarian Academy of Sciences, Hungary, Bulgaria, 1983.

[8] R. K. Thumbakara, “Intuitionistic Fuzzy Subframes," The Journal of Fuzzy Mathematics, vol. 15, no. 3, pp. 525-539, 2007.

[9] R. K. Thumbakara, "On intutionistic fuzzy filters of a frame," in Proceedings of the UGC sponsored National Seminar on Fuzzy mathematics and Applications, M.A. College, Kothamangalam, India, July 2005. 


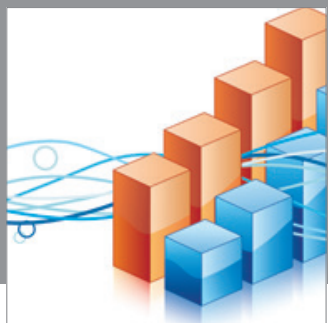

Advances in

Operations Research

mansans

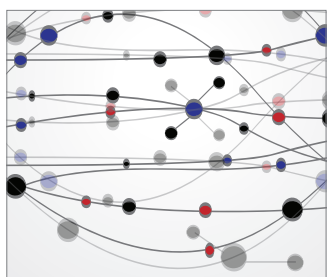

The Scientific World Journal
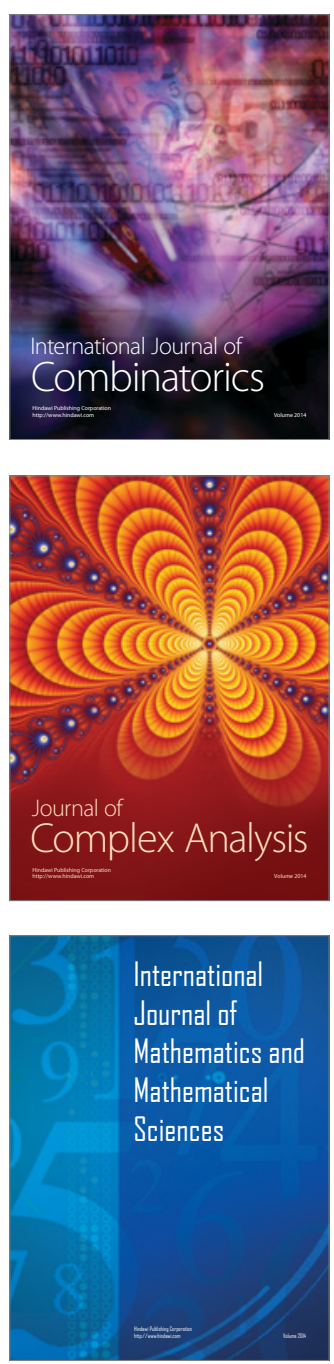
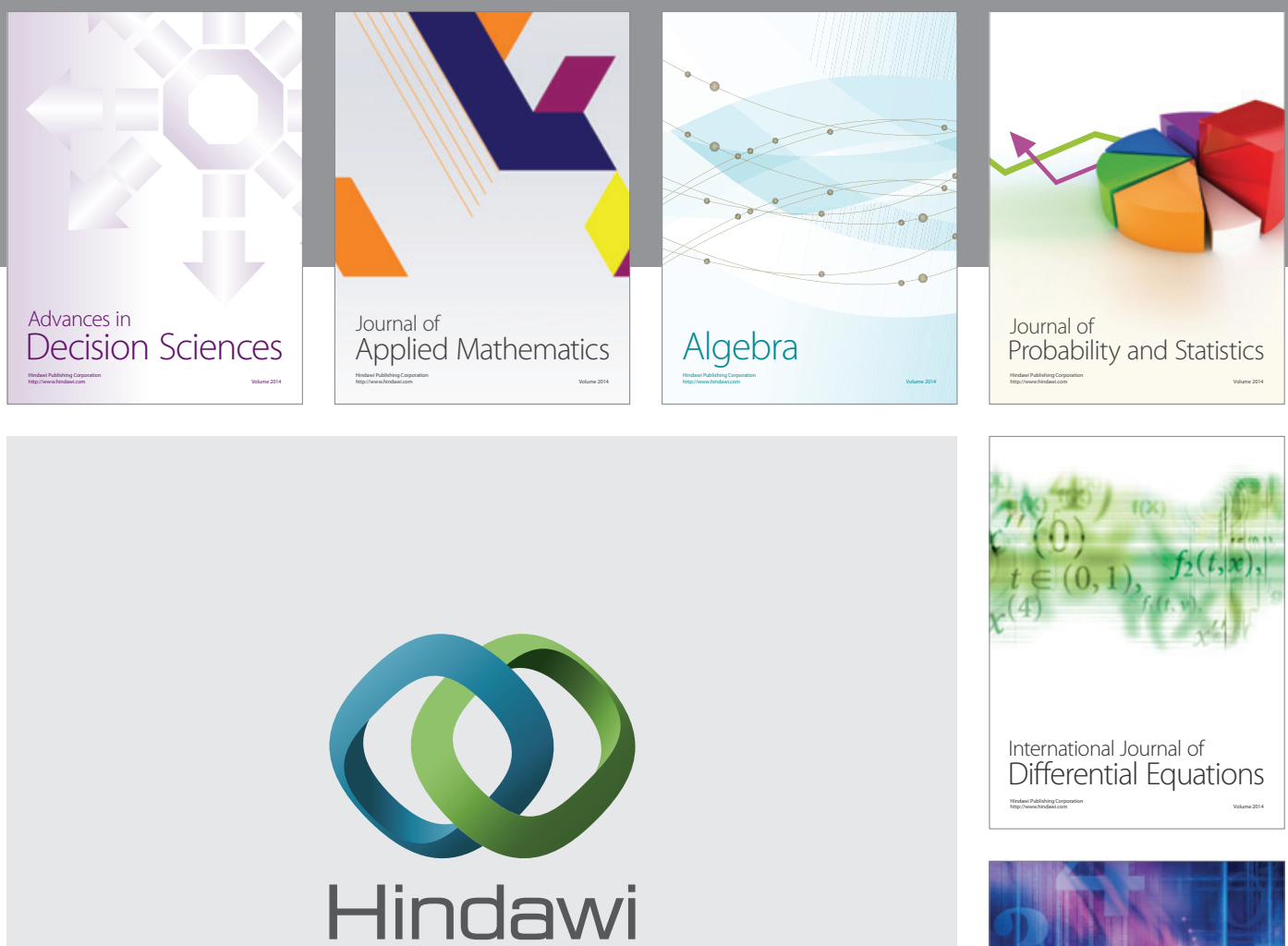

Submit your manuscripts at http://www.hindawi.com
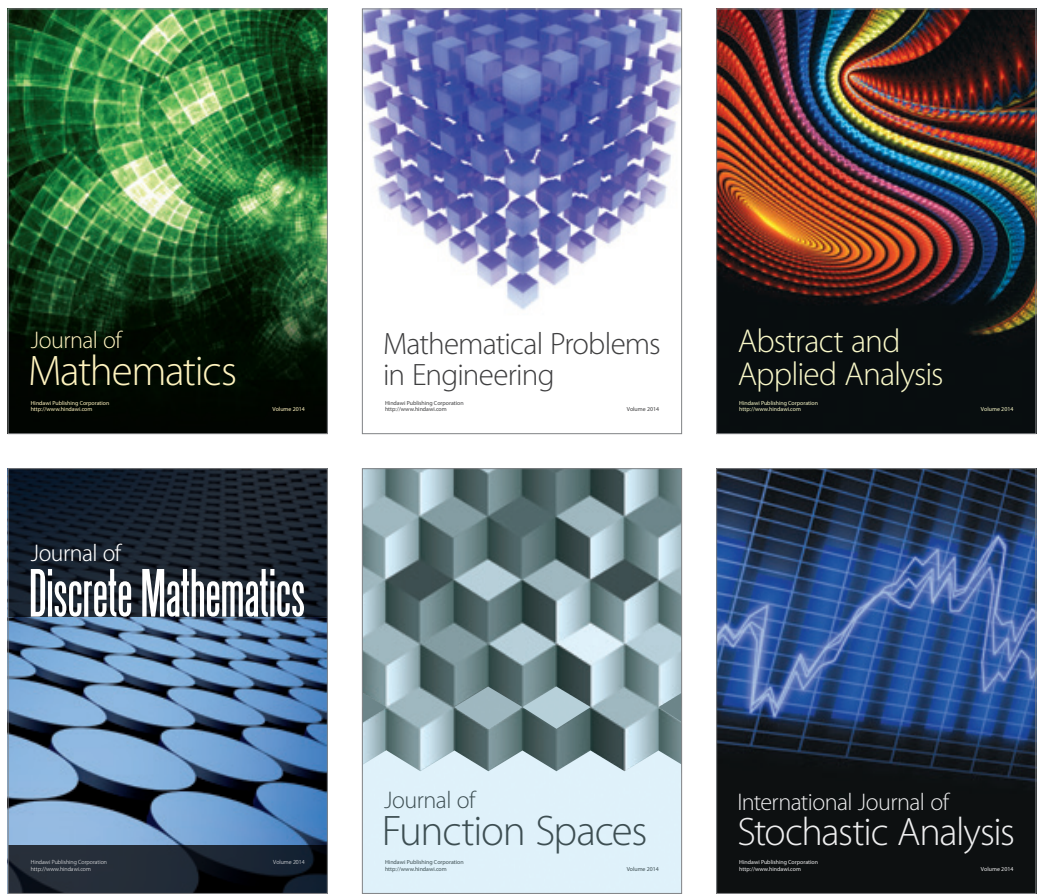

Journal of

Function Spaces

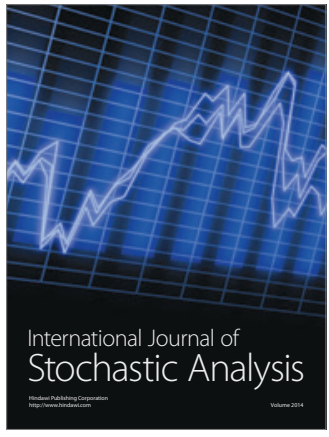

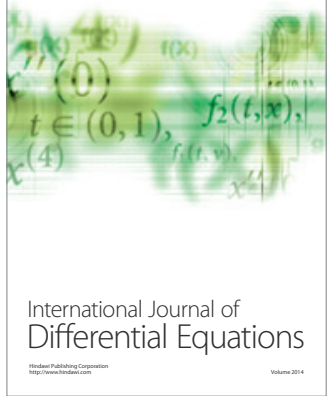
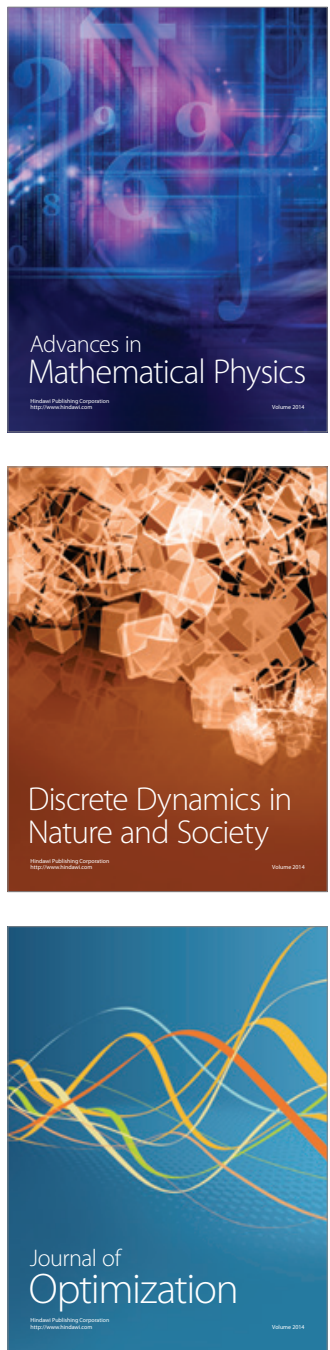